REVISTA CIENCIAS BIOMÉDICAS

HISTORIA DE LA MEDICINA EN CARTAGENA

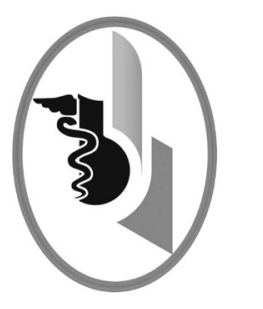

\title{
CARLOS CRUZ ECHEVERRÍA, MÉDICO Y ESCRITOR. SINOPSIS DE SUS DOS OBRAS LITERARIAS*
}

\author{
CARLOS CRUZ ECHEVERRÍA, DOCTOR AND WRITER: \\ TWO OF HIS LITERARY WORKS
}

Durán-Méndez Leidy ${ }^{1}$

\section{RESUMEN}

El doctor Carlos Cruz Echeverría fue egresado de la Facultad de Medicina de la Universidad de Cartagena y posteriormente, fue profesor de ella. Fue uno de los más importantes exponentes de la anatomía del sistema nervioso central y periférico que ha tenido dicha escuela médica. Hábil en el dibujo anatómico, ilustraba sus clases trazando con tizas de colores en el tablero los elementos principales de la neuroanatomía. Además cultivó la escritura de ficción, y por medio de dos libros de relatos dejó retratada a la escuela médica de la Universidad de Cartagena, así como a toda la sociedad en la cual dicha universidad estaba inmersa. En el presente texto se reproducen frases del escritor cartagenero Álvaro Angulo Bossa, quien en su libro Cartagena de Indias: novelistas y cronistas costumbristas, se expresó sobre las dos obra de ficción realizadas por Carlos Cruz Echeverría. Rev.cienc.biomed. 2016;7(1):157-163.

\section{PALABRAS CLAVE}

Historia de la Medicina; Educación Médica; Historiografía; Anatomía.

\section{SUMMARY}

Doctor Carlos Cruz Echeverría graduated from Medicine Department at Universidad de Cartagena where he was professor. He was one of the most important exponents of the central and peripheral nervous system anatomy that had had this department. He was skilled in anatomical drawing, he illustrated his classes by drawing with chalk on the board the main elements of neuroanatomy. He also cultivated fiction writing, and through two books of stories he portrayed the medical school of the University of Cartagena, as well as the whole society in which the university was immersed. In the present text are reproduced sentences of the writer Álvaro Angulo Bossa, who in his

Un producto del Semillero de Investigación Historiografía Médica Cartagenera (HISTORI-MED).

1 Estudiante. Facultad de Medicina. Semillero de Investigación de Historiografía Médica Cartagenera (HISTORI-MED), el cual hace parte de la Facultad de Medicina. Universidad de Cartagena-Colombia. 
book Cartagena de Indias: novelistas y cronistas costumbristas, expressed his opinion about the two fiction works written by Carlos Cruz Echeverría. Rev.cienc.biomed. 2016;7(1):157-163.

\section{KEYWORDS}

History of Medicine; Medial education; Historiography; Anatomy.

\section{INTRODUCCIÓN}

Carlos Cruz Echeverría egresó de la Universidad de Cartagena como médico, para posteriormente hacerse especialista en neurología. Fue de los primeros cultivadores de las neurociencias en la ciudad de Cartagena, destacado profesor de Neuroanatomía Humana y Neurología Clínica. $\mathrm{Ha}$ sido uno de los médicos cartageneros que retrató con pasión su entorno Caribe en libros de literatura de ficción con personajes inspirados o gestados en hombres y mujeres de su entorno, y nutridos del paisaje y de la realidad circundante. Desde muy joven su trasegar en la vida académica y profesional fue más allá del solo ejercicio de la profesión de médico, lo que le permitió dejar una importante huella en lo político, cultural y deportivo.

Fue columnista del Diario de la Costa, El Universal y de El Espectador/Costa, por más de 12 años. Político activo dentro del Partido Liberal colombiano y elegido en dos ocasiones como presidente del Concejo de la ciudad de Cartagena. Líder y dirigente deportivo, especialmente del boxeo; en este último escenario cumplió un importante papel, teniendo siempre a la mano los preceptos médicos y siendo pionero en el entorno de la Costa Norte colombiana, en lo que hoy se conoce como medicina deportiva.

En sus escritos de prensa siempre argumentó sus tesis y propuestas con el conocimiento médico del funcionamiento cerebral que poseía y con información actualizada referente a las vías de funcionamiento cerebral. Asimismo, estudió la fisiología y la patología de la cognición, el aprendizaje, el comportamiento humano y la esencia o naturaleza de la psiquis de los individuos, con lo que pudo matizar textos de aspecto político, social, cultural y gremial.

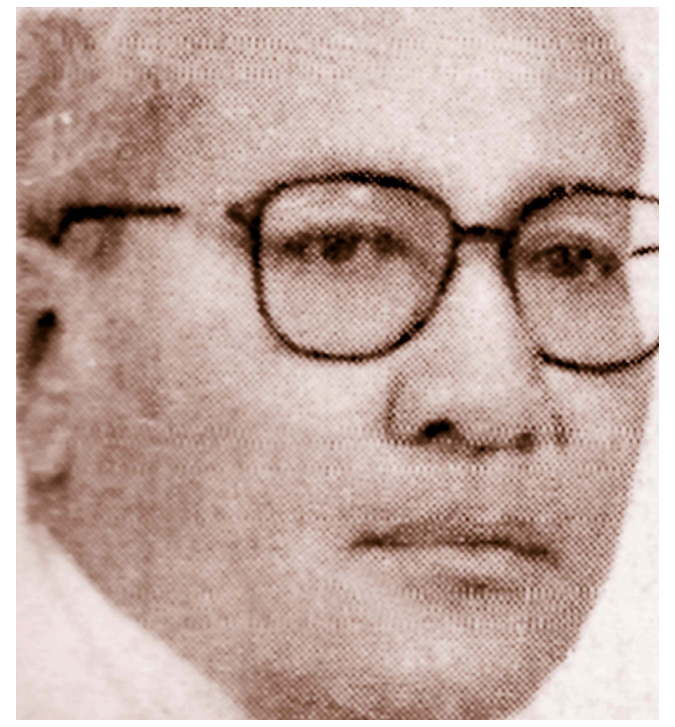

Carlos Cruz Echeverría

La Revista Ciencias Biomédicas y el Semillero de Investigación Historiografía Médica Cartagenera (HISTORI-MED) con el propósito de homenajear la memoria del profesor Carlos Cruz Echevarría, quien por más de veinte años fue profesor de Neuroanatomía y Neurofisiología, cultivador y creador de la catedra de lo que hoy se denomina neurociencias en la Universidad de Cartagena, reproduce apartes del texto del escritor cartagenero Álvaro Angulo Bossa en su libro Cartagena de Indias: novelistas y cronistas costumbristas, donde realizó un análisis de las dos obras literarias de ficción, escritas y publicadas por el doctor Carlos Cruz Echeverría. El primero dijo con respecto al profesor:

"Los siguientes párrafos a la memoria de mi amigo Carlos Cruz Echeverría, en agradecimiento de aquella dedicatoria escrita para mí en su libro Pasiones de biches y maduros, que a la letra dice: Para el extraordinario y original "Curro" 
Angulo Bossa. Es uno de mis personajes y algún día le haré un libro con sus ricas características humanas. (Fdo.) Carlos Cruz Echeverría, Cartagena, 28 de enero de 1994".

Carlos Cruz Echeverría escribió dos obras literarias: Pasiones de biches y maduros y Los asombros de Congobarato. Fue hijo de Miguel Cruz Olmos, hizo parte de una familia de clase media cartagenera, $y$, a través de la escritura de ficción dejó retratada la sociedad cartagenera de inicios de la segunda mitad del siglo $\mathrm{XX}$, de donde tomaba aspectos cotidianos, el desparpajo y el folclorismo del pueblo costeño. La medicina, la política y el deporte hicieron parte de sus dos obras.

"El verdadero valor de la obra de Cruz Echeverría radica en su estilo llano, sencillo y sin palabras rebuscadas. Hace gala de un excelente y picante humor con sabor Caribe, de donde fluyen a todo momento las ingenuas travesuras, similares a las de los chicos malos de las tiras cómicas, que nos hacen reír a carcajadas en medio de la lectura", dice Angulo Bossa.

Además de esos dos libros publicó en el Magazín Dominical del Periódico el Universal el 25 de agosto de 1996, el cuento titulado Efectos de un grillo enamorado, donde el personaje es un agobiado médico que tuvo que realizar una nocturna lucha contra el canto agudo y constante de un grillo en celo.

\section{PASIONES DE BICHES Y MADUROS}

Este pequeño y primer libro de Carlos Cruz Echeverría está conformado por dos relatos: el primero es una crónica sobre la vida cotidiana de Cartagena durante la primera mitad del siglo $X X$, editado en Litomar, sin fecha de impresión, pero se sospecha que debió ser en 1996 con prólogo de Manuel Zapata Olivella. El relato se refiere a la vida de la Facultad de Medicina de la Universidad de Cartagena con sus contados estudiantes, pues solo se permitía el ingreso de 50 de ellos para cursar el primer año, lo cual no era suficiente para satisfacer la necesidad de formar médicos para la región Caribe. Hubo muchas presiones de diferentes sectores de la sociedad, hasta que en 1946

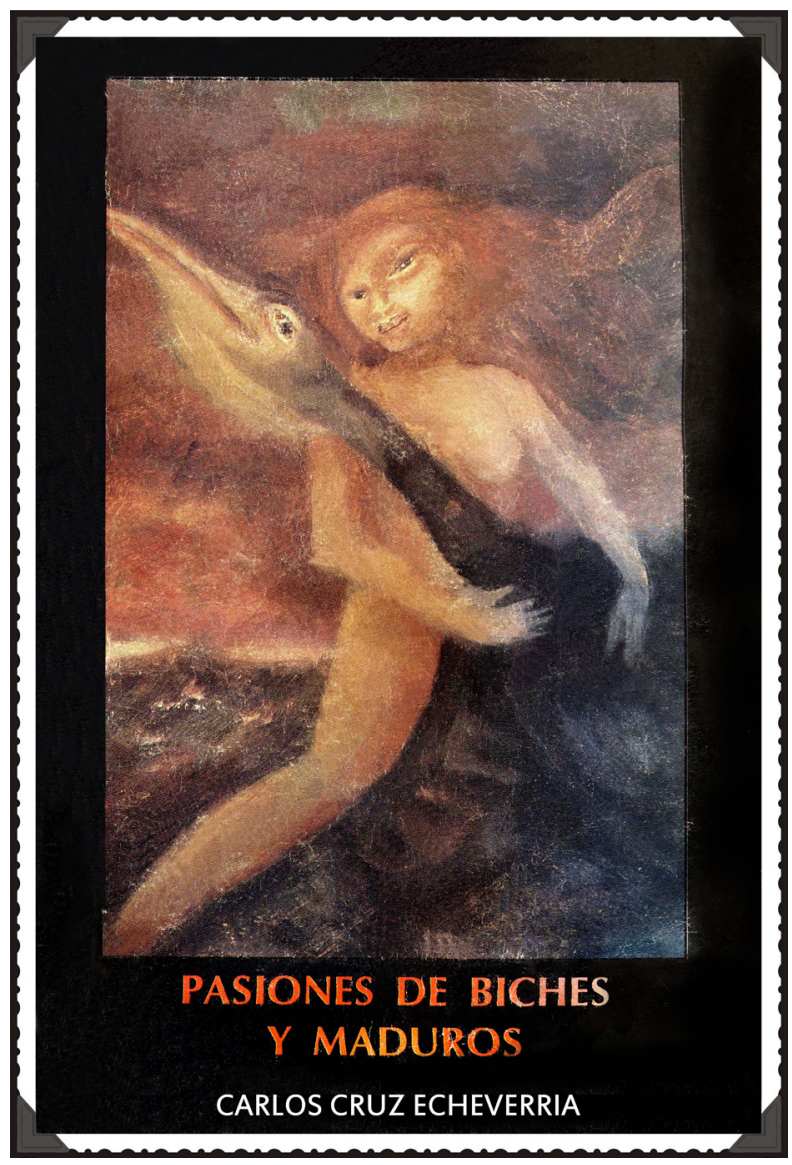

Caratula del libro. Dibujo Cheo Cruz.

se rompieron las barreras y se permitió la entrada a mayor número de estudiantes. Así las cosas, ante este aumento repentino, el gobierno departamental tuvo que duplicar el número de profesores y las instalaciones del viejo claustro de San Agustín tuvieron que ser remodeladas para albergar a la nueva población estudiantil. Cruz Echeverría dijo que:

\begin{abstract}
"Dentro del estudiantado había un gran número de perezosos, pero había excepciones como en el caso de Pablo Petrochelli Zunino, estudiante abstemio y aplicado, decente en el hablar e interesado por las investigaciones científicas. Según confesó el propio escritor, el personaje de Petroccelli fue inspirado en el estudiante Aníbal Perna Mazeo".
\end{abstract}

Que posteriormente llegaría a ser profesor de la Universidad de Cartagena. 
El narrador cuenta la vida apacible de los estudiantes de Medicina en el Corralito, la que transcurría en un permanente trasegar entre el claustro de San Agustín de la calle de la Universidad, el parque Fernández de Madrid en San Diego y el tradicional e histórico Hospital Santa Clara. En el parque, los alumnos se dedicaban, durante las noches de verano, a estudiar en grupos bajo los postes del alumbrado público. Los fines de semana, los estudiantes pobres empeñaban sus valiosos libros en los Montes de Piedad de la calle de la Media Luna, para financiar sus parrandas sabatinas, otros, recibían la ayuda de algunos compañeros de la clase media. Después de maratónicas parrandas, se dirigían en grupo a la fonda La Cueva, situada en el mercado público y al aire libre. Allí la suculenta cena, consistente en conejo ahumado, guartinaja, o gallina criolla con arroz de manteca.

La segunda parte de esta crónica trata sobre la aventura científica del graduando Petrocceli, quien solicitó al autor acompañarlo a realizar una investigación en Moñitos, una lejana población cerca de San Bernardo del Viento. El motivo de la investigación era probar en su tesis de grado, los efectos de la vacuna contra la histoplasmosis pulmonar, y la razón era que, desde Moñitos, había llegado al Hospital Santa Clara un niño que murió del mal. Deseaba Petroccelli realizar una tesis de elevado nivel y que fuese considerada tesis laureada, el distintivo de mayor reconocimiento.

"En el reloj público de la boca del puente, eran las 12 del día 29 de agosto de 1955, y desde allí salieron de viaje hacia Moñitos. La población que carecía de luz eléctrica y de agua potable. Las calles eran de arena. En aquel entonces allí no se conocía el pavimento $y$ al fondo del pueblo, las montañas cordobesas adornaban el firmamento. La comida era esencialmente carne de monte, pero también el pescado era abundante en aquel remanso de la naturaleza. El viaje hacia aquella población morena duró tres días en un espacioso motovelero accionado por un motor de camión, adaptado a la nave por sus dueños en Panamá. Durante el viaje, los investigadores se extasiaron con el paisaje marino caribeño y con la brisa tenue de agosto. Al penetrar en la inmensidad del mar Caribe, la motonave comenzó a deslizarse suavemente, subiendo y bajando al compás de las olas del mar profundo. Finalmente, al arribar al pueblo los recibió una nutrida comisión integrada por lo más selecto de la población: el Inspector de Policía, el Cura, los Morelos, el llamado Palomo y otros personajes importantes".

Al segundo día fue el de la vacunación y la toma de muestras, la cual es bellamente descrita y envuelta en una emotiva historia:

"Donde una hermosa mulata que llevaba a su hermanito de brazos para que lo vacunaran, deslumbró al casto Petrochelli, quien de inmediato se enamoró perdidamente. El narrador cuenta que en Moñitos era costumbre ancestral que los campesinos pobres vendieran a sus hijas y el comprador, por lo general era casi siempre un adinerado hacendado. Se urde una especia de pedida de mano, lo que en otras palabras significaba la compra de la joven. Petrochelli se opuso a comprar ron para el brindis, con el objeto de darle solemnidad al acto y el padre de la niña se opuso señalando que la muchacha no iba a servir porque estaba muy biche. Los contertulios en el pueblo atribuyeron el fracaso de la pedimenta a la falta de música y ron". Varias expresiones populares y un entorno cultural y mágico, tormentas y actos de brujería, se ciernen sobre la realización de la investigación y las consecuencias en los alcances históricos de la tesis.

Un par de días después los dos médicos investigadores regresaron a Cartagena. Carlos Cruz Echeverría cerró su relato diciendo:

"En los días siguientes, Pablo hizo las pruebas de laboratorio a las muestras recolectadas y confeccionó la ansiada tesis, que presentó al jurado con la esperanza de laureación. Extrañamente, los ilustres jueces, le dieron calificación común y corriente, como si se tratara de las tesis 
copiadas de los libracos de la biblioteca. No tuvieron en cuenta la originalidad y el esfuerzo, ni la demostración objetiva que en Moñitos existía un foco de infección terrible derivado de las condiciones insalubres, que era necesario combatir. La depresión del italiano fue prolongada y se dedicó a la oración y a exorcizar los pecados cometidos en el viaje".

Angulo Bossa refiere que al final del relato:

"El narrador cuenta que de repente apareció una bandada de alcatraces barrigudos y enormes y se posaron sobre la playa. Entre aquellos pelícanos estaba el alcatraz real, sobre el cual se montó la mulata y velozmente desapareció en el firmamento".

La segunda aventura de Pasiones biches $y$ maduros trata sobre el viaje a Cuba de la Fedebox colombiana para representar al país en un campeonato de boxeo. Esta historia no tiene el entorno médico pero sí otra actividad apasionante para Cruz Echeverría: el deporte. El presidente de la misión era el doctor Cocosolo, conocido abogado de la ciudad, nacido en Palenque y criado en Cartagena por el doctor Arístides Paz Viera, respetado médico y político liberal de la ciudad, quien lo tomó en adopción desde niño, lo mantuvo del todo a su lado y le dio educación, incluyendo la universitaria. Como miembro de su raza, Cocosolo era negrito como el carbón, simpático y de finos modales.

"La ideología de Cocosolo era de izquierda, casi revolucionaria, pero militaba en el partido liberal y lo representaba como diputado a la Asamblea, posición que le había alcanzado a través de intrigas políticas, El defecto de Cocosolo era la tacañería, ya que siempre pretendía comer, beber y comprar a costa de los demás. Los compañeros resolvieron hacerle una trastada en asocio de otro miembro de la misión: consiguieron un formulario del restaurante-bar del hotel habanero, y lo llenaron con una supuesta cuenta firmada por algunos miembros de la delegación, en especial por el serio y circunspecto entrenador de los boxeadores. La cuenta fue dirigida al presidente doctor Cocosolo, cuando la recibió casi sufre un desmayo pues ascendía, a mil dólares", dice Álvaro Angulo Bossa en su comentario.

"El Presidente se dirigió a reclamar al entrenador, pero este lo agarró por el cuello y casi lo noquea de un derechazo, gritándole furioso que él era un hombre abstemio y enemigo de la parranda. En la delegación se regó la bola del presunto lío de Cocosolo, de quien se decía quedaría a buen recaudo de la policía cubana para responder por la cuenta. Cocosolo se ausentó del hotel, pero al llegar el bus que los transportaría al aeropuerto, entró de primero y se escondió en el último asiento del automotor. Cuando iban a partir, el narrador cuenta que consiguieron que un barbudo miliciano entrara al colectivo y preguntara por Cocosolo, para decirle que debería ir a la recepción del hotel para saldar cuentas pendientes".

"En el final de la historia Cocosolo, asustado y apesadumbrado, se baja del bus y se dirige a la recepción con la intención de cumplir aquella orden de la autoridad. Pensaba que su caso crearía un conflicto internacional y vergonzoso para Colombia. Temblaba de miedo y quería esconder su rostro en el maletín que portaba. Pero al llegar a la recepción, y preguntar por la cuenta que tenía pendiente, el empleado le dijo: coño chico. Tú no debes nada. Esas son cabronadas de tus amigos".

Así culmina esta jocosa historia, con la cual se demuestra que Carlos Cruz Echeverría, aunque no era un depurado escritor, como afirma su prologuista, era realmente hábil con la sátira y el humor cartagenero. Carlos Cruz descolló como importante exponente del sabor cultural y del mestizaje propio de comunidades cartageneras que se desenvolvían en un medio lleno del calor tropical y la brisa Caribe. Este par de relatos cortos señalan su cosmovisión y su pensamiento dicharachero, la otra cara de la moneda del profesor y exigente académico médico de la neurología y las neurociencias. Ello era característica de la época médica que le tocó vivir, cuando el médico formado bajo la sentencia de que el médico que 
solo medicina sabía, ni medicina sabía, era en gran esencia culto y se destacaba en la sociedad por lo médico y por ser un exitoso cultivador de las artes, la plástica, el discurso político, la doctrina y el deporte.

\section{LOS ASOMBROS DE CONGOBARATO}

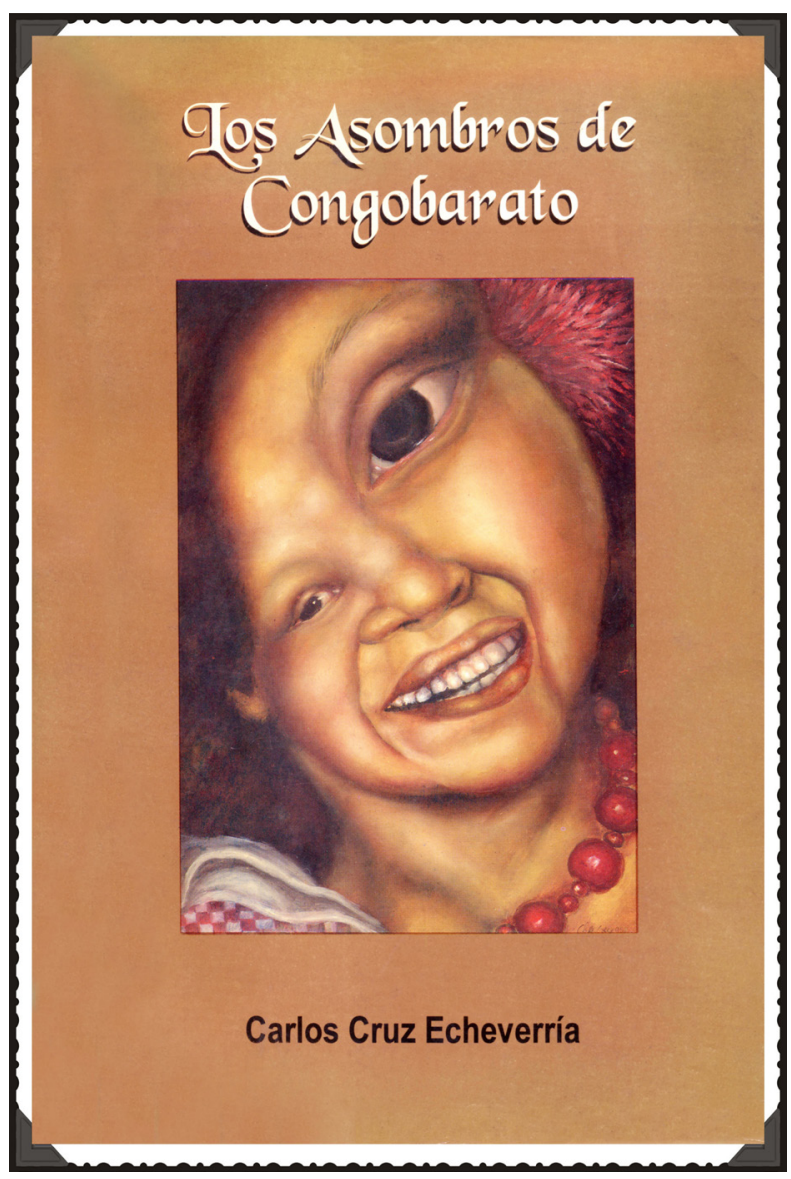

Caratula del libro: Ilustración Cheo Cruz.

Fue su segundo libro de ficción, también inspirado en la realidad de su entorno, en sus amigos, e incluso, en sus detractores políticos, a todos los caricaturizó con cariño y humor. Fue publicado en Editorial Lealon de Medellín, obra inscrita en la modalidad de novela en un concurso convocado por la Secretaría de Educación y Cultura Distrital cartagenera. Aunque no obtuvo el primer premio, el gobierno distrital ordenó su publicación, teniendo en cuenta su valor costumbrista respecto a la región y la manera como relataba las fiestas religiosas y paganas.
"El prólogo de la obra fue realizado por Manuel Zapata Olivella, reconocido e inolvidable escritor cordobés. Zapata Olivella explica que Cruz Echeverría actúa en el relato no corrigiendo entuertos, como Don Quijote, simplemente, para él, Carlos se cubre la cara con una capa como Quevedo, se mezcla con los actores en el mundillo del chisme, señala sus defectos y se burla de ellos. Finalmente, satiriza las costumbres políticas de la costa, no para corregirlas sino para criticarlas jocosamente", dice Angulo Bossa.

Geográficamente, el relato novelado de Carlos Cruz Echeverría ocurre supuestamente entre Cartagena y una población exuberante, frente al mar, rodeada de arroyuelos y también de una vegetación de hermosos árboles de estirpe marina, a la cual él llama Congobarato. La obra es intemporal, Ilena de personajes de ficción que son retratos calcados de personalidades de Cartagena. A lo largo de la novela surgen señales de ciertos hechos que sucedieron en la ciudad durante la segunda mitad del siglo XX. Un ejemplo fue el famoso mordisco con el que el doctor Juan Arango, dinámico y folclórico Alcalde Mayor de Cartagena, le cercenó la oreja a un turista. Este profesional fue médico de amplia experiencia en oftalmología, escritor de ficción con sensible labor profesional en la comunidad cartagenera y conocido popularmente como Juancho Arango. El libro se antoja como su nombre, un caudal de hechos asombrosos.

"La narración de la historia es de tipo lineal, y el género al cual pertenece este relato novelado es el costumbrista, obviamente, como dice Manuel Zapata Olivella, a todo lo largo del relato se percibe y se siente un tono burlón y satírico, tal como acostumbraba Quevedo en sus escritos, quien se sirvió de la burla y de la sátira para mostrar su crítica al mundo que le rodeaba. Rufino Lobo Sánchez es el personaje central de esta novela costumbrista. Era Jefe político de la capital del Caribe, una ciudad colonial situada a orillas del mar, con todas las trazas de ser Cartagena, y quien aspiraba a ser elegido para ocupar importantes posiciones de representación política. 
Lobo Sánchez era un inteligente abogado y habilidoso político, que a todas horas se hallaba en campaña electoral para conseguir votos a través de los gamonales de ciertos pueblos de la Costa, y en especial de los terratenientes y politiqueros de Congobarato. Al momento de pronunciar sus discursos, era revolucionario a carta cabal pero después de elegido cambiaba de actitud".

Otros personajes presentes en Los asombros de Congobarato: el experto tinterillo y poeta, un tegua que había comenzado su vida como camillero en el hospital colonial de la capital, de seguro el llamado Hospital Santa Clara de San Diego, Tiburcio que curaba a los enfermos con un rayito de luz, un teniente corpulento y malgeniado que había soñado ser el autor del asesinato de su madre y un trabajador del terminal marítimo, quien había trabajado en Nueva York como estibador de los muelles de Brooklyn. Un amplio zoológico de personalidades, excentricidades y desmanes, pero que son vistos como la realidad simple y diáfana. Todos ellos se mezclan en un ambiente tragicómico donde el mundo político está centrado en favores y votos de campaña.

Álvaro Angulo Bossa dice: "Este libro de Carlos Cruz Echeverría, tal como señala el prologuista Manuel Zapata Olivella, trata sobre el reino tropical de Congobarato, un simulacro de democracia, donde cada quien se sentía libre, aunque viviera encadenado al subdesarrollo, la pobreza y la ignorancia. Corralejas de toros, cumbiambas y fandangos, reinados de asnos, comicios fraudulentos, cantinas para emborracharse y hamacas para reposar".

En Congobarato se observa una vida llevada adelante con naturalidad y sin aspavientos, donde los eventos desmedidos son productos de una esencia cultural deslumbrante, donde sin sobresaltos se realiza cacería de cangrejos azules, se observan dirigibles con prisioneros en el interior, los gringos bailan sin acierto en las cumbiambas, donde existen cacerías de patos por las ciénagas del Canal del Dique y donde las fiestas tienen duraciones kilométricas.

Carlos Cruz Echeverría "hace paladear un carnaval, donde la tragedia se encubre con mascarones burlescos", dijo en su texto Angulo Bossa. En el texto la medicina y los médicos no podían estar ausentes. Pócimas y prescripciones son hechas. Explicaciones desde la neurología y la psiquiatría en el lenguaje popular o desde la imaginación ilimitada del Caribe asaltan al lector en algunos rincones.

Carlos Cruz Echevarría con dos relatos y una novela dejó una huella importante: señaló con detalle aspectos del costumbrismo cartagenero mientras plasmaba la vida, el trasegar y el desenvolvimiento de una escuela médica, sus estudiantes y sus egresados que se encargaron de hacer más desbordada la realidad del Caribe colombiano.

\section{LECTURAS RECOMENDADAS}

1. Angulo-Bossa A. Cartagena de Indias: novelistas y cronistas costumbristas. http:// academiadelahistoriadecartagenadeindias.org/Publicaciones/Articulos\%20Alvaro\%20 Angulo\%20Bossa/NovelistasYCronistasCostumbristas.pdf.

2. Cruz-Echeverría C. Los asombros de Congobarato. Editorial LeaLon. Medellín. 1996.

3. Cruz Echeverría C. Pasiones de biches y maduros. Impresión Litomar. Cartagena.

4. Cruz Echeverría C. Efectos de un grillo enamorado. Magazín Dominical del Periódico el Universal el 25 de agosto de 1996. 